\title{
Depression and Somatic Symptoms May Influence on Chronic Prostatitis/Chronic Pelvic Pain Syndrome: A Preliminary Study
}

\author{
Jun Sung Koh ${ }^{1}$, Hyo Jung Ko², Sheng-Min Wang ${ }^{3}$, Kang Joon Cho', \\ Joon Chul Kim¹, Soo-Jung Lee ${ }^{3}$, and Chi-Un Pae ${ }^{3,4} \bowtie$ \\ ${ }^{1}$ Department of Urology, The Catholic University of Korea College of Medicine, Seoul, Republic of Korea \\ ${ }^{2}$ Department of Psychiatry, Seoul Metropolitan Eunpyeong Hospital, Seoul, Republic of Korea \\ ${ }^{3}$ Department of Psychiatry, The Catholic University of Korea College of Medicine, Seoul, Republic of Korea \\ ${ }^{4}$ Department of Psychiatry and Behavioral Sciences, Duke University Medical Center, Duram, NC, USA
}

The present study is the first one to investigate the impacts of depression and somatization on the disease severity and quality of life (QoL) in patients with chronic prostatitis/chronic pelvic pain syndrome (CP/CPPS). The Korean version of National Institutes of Health (NIH)- Chronic Prostatitis Symptom Index (CPSI) for severity of CP/CPPS. Korean version of Patient Health Questionnaire-9 (PHQ-9) for depression, Korean version of Patient Health Questionnaire-15 (PHQ-15) for somatization, and Korean version of EuroQol Questionnaire-5 Dimensions (EQ-5D)- [(EQ-5D utility index and visual analog scale (EQ-5D VAS)] for QoL, were administered. Eighty patients were enrolled. The NIH-CPSI total scores were significantly higher in those with depression $(25.3 \%, \mathrm{p}=0.01)$ or somatization $(23.2 \%, \mathrm{p}=0.03)$ than in those without. These trends toward significantly negative influence of depression and somatic symptoms on QoL were also observed. Our preliminary results indicate that depression and somatization may have negative influence on the symptom severity and QoL in patients with CP/CPPS. However, adequately-powered and more well-designed studies are mandatory to prove our results.

Psychiatry Investig 2014;11(4):495-498

Key Words Chronic prostatitis/chronic pelvic pain syndrome, Depression, Somatization.

\section{INTRODUCTION}

Chronic prostatitis/chronic pelvic pain syndrome $(\mathrm{CP} /$ CPPS) is a chronic pain disorder characterized by the presence of noninfectious pelvic or perineal pain lasting longer than 3 months. Its diagnosis is based on criteria suggested by the International Prostatitis Collaborative Network of the National Institutes of Health (IPCN-NIH). ${ }^{1,2}$

However, the exact pathophysiology and mechanical disturbances underlying CP/CPPS remain elusive, and this syndrome is now considered to be a multifactorial medical condition that includes psychological aspects and requires a multimodal treatment approach. ${ }^{3}$

Received: July 27, 2013 Revised: September 9, 2013

Accepted: September 12, 2013 Available online: October 20, 2014

$\triangle$ Correspondence: Chi-Un Pae, MD

Department of Psychiatry, Bucheon St. Mary's Hospital, The Catholic University of Korea College of Medicine, 327 Sosa-ro, Wonmi-gu, Bucheon 420-717, Republic of Korea

Tel: +82-32-340-7067, Fax: +82-32-340-2255, E-mail: pae@catholic.ac.kr

(a) This is an Open Access article distributed under the terms of the Creative Commons Attribution Non-Commercial License (http://creativecommons.org/licenses/by$\mathrm{nc} / 3.0$ ) which permits unrestricted non-commercial use, distribution, and reproduction in any medium, provided the original work is properly cited.
According to a number of epidemiological studies using different methodologies, the prevalence of CP/CPPS varies from approximately from 8 to $20 \%$ worldwide. ${ }^{4-7}$ Its natural history has not been intensively studied, but it has been proposed that chronic, persistent, or recurrent symptoms resulting in functional impairments are common in patients with CP/CPPS. ${ }^{8}$ Additionally, previous pain episodes and more severe symptoms are known to be high risk factors for chronicity. ${ }^{8}$

Although recent studies have found psychiatric symptoms, such as depression, to be one of the major contributing factors to CP/CPPS, a causal relationship between depression and CP/CPPS has yet to be clearly elucidated.

Most studies that have found a role for depression in $\mathrm{CP} /$ CPPS were conducted in Western populations; there is a dearth of data from Asian patients. Hence, the present study aimed to investigate the associations of depression and somatization with symptom severity in patients with CP/CPPS in a routine clinical practice to better understand the relationships between depression and somatization and CP/CPPS. 


\section{METHODS}

\section{Subjects and recruitment procedure}

Male participants were recruited at an outpatient clinic for prostatitis in the Department of Urology at Bucheon St. Mary's Hospital in Bucheon, Gyeonggi-Do, Korea. The recruitment period lasted from April 2011 to March 2012. Of the 324 patients interviewed, 80 male patients agreed to be enrolled in the study. The diagnosis of CP/CPPS was based on National Institutes of Health $(\mathrm{NIH})$ consensus $^{2}$ following a careful medical interview, digital rectal examination, urinalysis, and prostate secretion examination after prostate massage or urinalysis after prostate massage. Patients who exhibited pyuria and other genitourinary symptoms that may be associated with benign prostatic hyperplasia, neurogenic bladder, or genitourinary diseases other than CP/CPPS were excluded from the study to maximize diagnostic validity of CP/CPPS. The protocol of the present study was approved by the Institutional Review Board of the hospital (HC11EISE0009).

\section{Rating scales}

This study used the following measures to investigate associations between the presence of depression or somatization and symptom severity and quality of life (QoL) in CP/CPPS patients: the NIH-Chronic Prostatitis Symptom Index (NIHCPSI) to assess the severity of CP/CPPS symptoms, ${ }^{2}$ the Patient Health Questionnaire-9 (PHQ-9) to evaluate depression, ${ }^{9}$ the Patient Health Questionnaire-15 (PHQ-15) to determine somatization, ${ }^{10}$ and the EuroQol, ${ }^{11}$ specifically the EQ-5D utility index and EQ-5D visual analog scale (VAS), to measure QoL. All rating scales were the Korean version of the original scales and were administered by the treating physician or the patients themselves as appropriate.

\section{Statistical analysis}

Descriptive statistics for the baseline demographic and clinical variables of the participants $(n=80)$ are reported. For the analysis of differences in total scores on the NHI-CPSI, EQ5D utility index, and EQ-5D VAS according to the presence or absence of depression (defined by PHQ- 9 total scores $\geq 5)^{12}$ or somatization (defined by PHQ-15 total scores $\geq 5$ ), ${ }^{13}$ an analysis of covariance (ANCOVA) adjusted for age, first onset of symptoms, and duration of disease was conducted. For the analysis of categorical variables, a chi-square or Fisher's exact test was performed as appropriate. Odds ratios (ORs) with 95\% confidence intervals (CIs) were also calculated for the comparison of severity according to classifications based on NIH-CPSI total score between those with and without depression and those with and without somatization. Spearman's correlation analysis was performed to evaluate the associations among baseline individual total scores from the NIH-CPSI, PHQ-9, and PHQ-15. Furthermore, logistic regression analysis was used to identify predictors (independent variables: age, duration of disease, first onset, baseline total scores on PHQ-9 and PHQ-15, EQ-5D score, EQ-5D VAS score, and presence or absence of depression or somatization) of the severity of CP/CPPS as classified by the NIH-CPSI total score (mild versus moderate/severe).

No adjustment for multiple comparisons was performed because the current sample size was small, and a Bonferroni correction would increase the likelihood of a type II error and publication bias. With these statistical parameters, the power of the sample was 0.6249 after adjusting covariates to detect a medium to large effect size (Cohen's $\mathrm{d}=0.6$ ) which corresponded to a difference of 4.8 on the NIH-CPSI total score between those with and without depression. Statistical significance was set at $\mathrm{p}<0.05$, two-tailed, for all comparisons. Statistical analyses were conducted using NCSS $2007^{\circledR}$ Power Analysis \& Sample Size Software (Kaysville, Utah, USA).

\section{RESULTS}

The mean ( \pm standard deviation) age and duration of disease were $50.1 \pm 12.2$ years and $15.8 \pm 21.1$ months, respectively. Of the participants, $82.5 \%(\mathrm{n}=66)$ were married, $31.3 \%$ (25) were new-onset patients, $46.5 \%$ (37) had graduated from university, $81.3 \%$ were employed, and $57.5 \%$ (45) had medical comorbidities.

Table 1 summarizes the results of the study. The mean total score on the NIH-CPSI indicates moderate tosevere CP/ CPPS among these patients. Mean scores on the PHQ-9 and PHQ-15 were 4.6 [ $(\mathrm{SD}=4.5,95 \% \mathrm{CI}=3.6-5.6 ; 53.8 \%$ normal to minimal depression ( $\mathrm{n}=43$ ); $46.2 \%$ mild to severe depression $(n=37)]$ and $5.5[(\mathrm{SD}=3.4,95 \% \mathrm{CI}=4.7-6.2 ; 42.5 \%$ normal to minimal somatic symptoms $(n=34) ; 57.5 \%$ low to severe somatic symptoms $(n=46)]$, respectively. This suggests that CP/CPPS patients may suffer from a broad range of clinical manifestations of depression, including somatization; physicians should be aware of this possibility (Table 1). Furthermore, NIH-CPSI total scores were significantly higher in those with depression (25.3\%) or somatization (23.2\%) than in those without.

The severity of CP/CPPS as defined by total points on the NIH-CPSI, the proportions of mild, moderate, and severe CP/CPPS symptoms were significantly different between those with and those without depression. These differences showed less severe symptoms among those without depression relative to those with depression; no differences in symptom severity were observed between those with and without somatization (Table 1). Similarly, the severity of CP/CPPS 
Table 1. Summary of the study results

\begin{tabular}{|c|c|c|c|c|c|}
\hline Parameters & $\begin{array}{l}\text { With depression } \\
\qquad(\mathrm{N}=37)\end{array}$ & $\begin{array}{l}\text { Without depression } \\
\qquad(\mathrm{N}=43)\end{array}$ & $\begin{array}{l}\text { With somatization } \\
\qquad(\mathrm{N}=47)\end{array}$ & $\begin{array}{l}\text { Without somatization } \\
\qquad(\mathrm{N}=33)\end{array}$ & $\begin{array}{c}\text { Total } \\
(\mathrm{N}=80)\end{array}$ \\
\hline NIH-CPSI & $23.6(7.2)$ & $18.8(9.4)^{*}$ & $22.8(7.9)$ & $18.5(9.3)^{\dagger}$ & $21.1(8.7)$ \\
\hline \multicolumn{6}{|l|}{ Severity by NIH-CPSI } \\
\hline Mild (0-14) & $3(8.1)$ & $17(39.5)$ & $8(17.0)$ & $12(36.4)$ & $20(25.0)$ \\
\hline Moderate (15-29) & $27(73.0)$ & $18(41.9)$ & $28(59.6)$ & $17(51.5)$ & $45(56.3)$ \\
\hline \multirow[t]{2}{*}{ Severe $(30-43)$} & 7 (18.9) & $8(18.6)$ & $11(23.4)$ & $4(12.1)$ & $15(18.8)$ \\
\hline & & $\chi^{2}=11.28, p=0.004$ & & $\chi^{2}=4.442, p=0.109$ & \\
\hline PHQ-9 & $8.2(4.1)$ & $1.4(1.3)^{\ddagger}$ & $5.6(4.9)$ & $3.1(3.4)^{*}$ & $4.6(4.5)$ \\
\hline PHQ-15 & $7.4(3.5)$ & $3.9(2.3)^{\ddagger}$ & $7.6(2.7)$ & $2.4(1.1)^{\ddagger}$ & $5.5(3.4)$ \\
\hline EQ-5D index values & $0.71(0.12)$ & $0.84(0.13)^{\ddagger}$ & $0.75(0.12)$ & $0.82(0.15)^{\S}$ & $0.78(0.02)$ \\
\hline EQ-VAS & $60.1(18.2)$ & $71.2(13.3)^{\|}$ & $61.9(16.3)$ & $72.0(15.3)^{\pi}$ & $66.1(16.6)$ \\
\hline
\end{tabular}

${ }^{*} \mathrm{p}=0.01,{ }^{\dagger} \mathrm{p}=0.03,{ }^{\ddagger} \mathrm{p}<0.0001,{ }^{\S} \mathrm{p}=0.039,{ }^{1 "} \mathrm{p}=0.002,{ }^{\mathrm{q}} \mathrm{p}=0.0067$. Mild vs. moderate plus severe, $\mathrm{p}=0.002$, odds ratio $(\mathrm{OR})=7.4,95 \%$ confidence intervals (CIs) $=1.765-35.833$ in those with depression, mild vs. moderate plus severe, $\mathrm{p}=0.067, \mathrm{OR}=2.8,95 \%$ CIs $=0.880-8.984$ in those with somatization, all comparisons are done between patients with or without depression ( 5 or more in PHQ- 9 total score) or somatization ( 5 or more in PHQ-15 total score), data represent mean (standard deviation) or number (\%). NIH-CPSI: National Institutes of Health-Chronic Prostatitis Symptom Index, PHQ-9: Patient Health Questionnaire-9, PHQ-15: Patient Health Questionnaire-15, EQ-5D: EuroQol-5 dimension, EQ-5D VAS: EuroQol-5 dimension Visual Analog Scale

symptoms, classified as mild versus moderate/severe according to NIH-CPSI scores, was greater among those with than those without depression ( $\mathrm{OR}=7.4, \mathrm{p}=0.002)$; no such difference was found between those with and without somatization (OR=2.8, $\mathrm{p}=0.067$ ) (Table 1). Trends toward a significantly negative influence of depression and somatic symptoms on QoL as measured by the EQ-5D utility index and the EQ-5D VAS were also observed (Table 1).

Significant correlations of total scores on the NIH-CPSI with PHQ-9 ( $\mathrm{r}=0.384, \mathrm{p}=0.0004)$ and PHQ-15 ( $\mathrm{r}=0.306, \mathrm{p}=$ $0.006)$ total scores were observed, as well as a correlation between PHQ-9 and PHQ-15 total scores ( $r=0.625$, $\mathrm{p}<0.0001)$. A logistic regression analysis revealed that total score on the PHQ-9 may be a potential predictor of the severity of $\mathrm{CP} /$ CPPS symptoms [Beta $=-0.449, \operatorname{Exp}(B)=0.638, p=0.03]$.

\section{DISCUSSION}

These preliminary data clearly demonstrate that a significant portion of CP/CPPS patients may struggle with symptoms of depression and somatization during the clinical course of their disorder. To the best of our knowledge, these are the first data supporting the substantial influence of depression and somatization on the severity of CP/CPPS. These results may help clinicians understand the role of depression and somatization as a potential moderator in the clinical manifestation of CP/CPPS.

A recent cohort study $(n=174)^{14}$ found that approximately $12 \%$ of all patients meet the criteria for any form of depressive disorder, whereas $18 \%$ of CP/CPPS patients are already being treated with various psychotropics for anxiety, depression, or stress. According to a recent NIH-sponsored CP cohort study $(n=463),{ }^{15}$ the severity of pelvic pain was strongly predicted by depressive symptoms, regardless of urinary symptoms and age. Additionally, another recent very large cohort study $(n=18,306)^{16}$ found that $163(5.34 \%)$ subjects in the CP/CPPS group and 494 subjects (3.24\%) in the control group received a diagnosis of depression during the 3-year follow-up period, indicating increased risk for developing depression in CP patients during the natural clinical course of their disorder. Furthermore, other evidence suggests that antidepressants may be effective for the treatment of CP/CPPS. ${ }^{17}$

As mentioned, the present data support previous Western and Asian studies that found that depression may be involved in the development and clinical course of CP/CPPS. ${ }^{14-16,18,19}$ In fact, depression and CP/CPPS may share, at least in part, several common pathophysiological mechanisms including the potential presence of a psychological initiator, hypothalamicpituitary-adrenal (HPA) axis alteration, inflammation, a serial cascade of events in the neuroendocrine system, central nervous system (CNS) sensitization (especially in relation to pain sensation), multifactorial CNS modulation, the involvement of oxidative stress, and the effects of antidepressants. ${ }^{3,20}$

In the present study, those with depression showed a significantly greater risk for severe forms of CP/CPPS (seven times higher) and somatization (three times higher) compared with those without depression or somatization, and total score of the PHQ-9 was found to be a potential predictor of the severity of CP/CPPS. Given these findings, depression may be interactively involved in the clinical manifestation of CP/CPPS. 
It has been also shown that somatization may lead to significantly lower satisfaction in certain domains of life; in fact, some studies have suggested that patients with CP/CPPS may struggle with various somatic symptoms. ${ }^{21}$ However, when considering all findings from the current investigation, somatization was found to contribute less to the symptoms of CP/ CPPS relative to depression. In future studies, the explicit roles of depression and somatization in CP/CPPS should be further explored.

There are a number of methodological limitations in this study including a small sample size, of the failure to consider other psychiatric comorbidities, the absence of control groups, and the lack of a formal diagnosis of depression by structured interview. ${ }^{22}$ Despite the small sample size, however, the actual power and effect size in the present sample (based on differences in NIH-CPSI total score between those with and without depression) was relatively satisfactory, reflecting the strong impact of depression on CP/CPPS severity.

Based on these preliminary results, the study methodology will need to be improved. The sample size should be sufficiently increased to detect minor but attributable factors. Anecdotal studies have consistently suggested that multiple psychological factors are involved in the development and clinical course of CP/CPPS, and thus, psychological factors other than depression should be included in future studies. Followup studies to detect the time effects of psychological factors on CP/CPPS are also warranted. Finally, the relationship between psychological factors and treatment response to $\mathrm{CP} /$ CPPS interventions would also be intriguing. Classification studies of CP/CPPS during screening or the initial evaluation stage based on the influence of psychological factors will also help clinicians identify characteristics that will lead to strategies and tactics to manage such patients in clinical practice.

Adequately powered and better-designed studies are necessary to better understand and clarify the precise role of depression and somatic symptoms on the development, clinical course, and treatment outcomes of CP/CPPS.

\section{Acknowledgments}

This study was partially funded by the Ministry of Health and Welfare, Korea (HI12C0003) and by Ildong Pharmaceutical.

\section{REFERENCES}

1. Mahal BA, Cohen JM, Allsop SA, Moore JB, Bhai SF, Inverso G, et al. The role of phenotyping in chronic prostatitis/chronic pelvic pain syndrome. Curr Urol Rep 2011;12:297-303.

2. Krieger JN, Nyberg L Jr, Nickel JC. NIH consensus definition and classification of prostatitis. JAMA 1999;282:236-237.

3. Nickel JC, Shoskes DA, Wagenlehner FM. Management of chronic prostatitis/chronic pelvic pain syndrome (CP/CPPS): the studies, the evidence, and the impact. World J Urol 2013;31:747-753.

4. Roberts RO, Lieber MM, Rhodes T, Girman CJ, Bostwick DG, Jacobsen SJ. Prevalence of a physician-assigned diagnosis of prostatitis: the Olmsted County Study of Urinary Symptoms and Health Status Among Men. Urology 1998;51:578-584.

5. Liang CZ, Li HJ, Wang ZP, Xing JP, Hu WL, Zhang TF, et al. The prevalence of prostatitis-like symptoms in China. J Urol 2009;182:558-563.

6. Ejike CE, Ezeanyika LU. Prevalence of chronic prostatitis symptoms in a randomly surveyed adult population of urban-community-dwelling Nigerian males. Int J Urol 2008;15:340-343.

7. Clemens JQ, Meenan RT, O’Keeffe Rosetti MC, Kimes T, Calhoun EA. Prevalence of and risk factors for prostatitis: population based assessment using physician assigned diagnoses. J Urol 2007;178:1333-1337.

8. Turner JA, Ciol MA, Von Korff M, Berger R. Prognosis of patients with new prostatitis/pelvic pain syndrome episodes. J Urol 2004;172:538541.

9. Han C, Jo SA, Kwak JH, Pae CU, Steffens D, Jo I, et al. Validation of the Patient Health Questionnaire-9 Korean version in the elderly population: the Ansan Geriatric study. Compr Psychiatry 2008;49:218-223.

10. Han C, Pae CU, Patkar AA, Masand PS, Kim KW, Joe SH, et al. Psychometric properties of the Patient Health Questionnaire-15 (PHQ-15) for measuring the somatic symptoms of psychiatric outpatients. Psychosomatics 2009;50:580-585.

11. EuroQol group. EQ-5D. Available at http://www.euroqol.org/euroqolgroup/board.html. Accessed on July 10, 2013

12. Kroenke K, Spitzer RL, Williams JB. The PHQ-9: validity of a brief depression severity measure. J Gen Intern Med 2001;16:606-613.

13. Kroenke K, Spitzer RL, Williams JB. The PHQ-15: validity of a new measure for evaluating the severity of somatic symptoms. Psychosom Med 2002;64:258-266.

14. Clemens JQ, Brown SO, Calhoun EA. Mental health diagnoses in patients with interstitial cystitis/painful bladder syndrome and chronic prostatitis/chronic pelvic pain syndrome: a case/control study. J Urol 2008;180:1378-1382.

15. Tripp DA, Curtis Nickel J, Landis JR, Wang YL, Knauss JS, CPCRN Study Group. Predictors of quality of life and pain in chronic prostatitis/chronic pelvic pain syndrome: findings from the National Institutes of Health Chronic Prostatitis Cohort Study. BJU Int 2004;94:1279-1282.

16. Chung SD, Huang CC, Lin HC. Chronic prostatitis and depressive disorder: a three year population-based study. J Affect Disord 2011;134: 404-409.

17. Xia D, Wang P, Chen J, Wang S, Jiang H. Fluoxetine ameliorates symptoms of refractory chronic prostatitis/chronic pelvic pain syndrome. Chin Med J (Engl) 2011;124:2158-2161.

18. Ahn SG, Kim SH, Chung KI, Park KS, Cho SY, Kim HW. Depression, anxiety, stress perception, and coping strategies in korean military patients with chronic prostatitis/chronic pelvic pain syndrome. Korean J Urol 2012;53:643-648.

19. Zhang GX, Bai WJ, Xu T, Wang XF. A preliminary evaluation of the psychometric profiles in Chinese men with chronic prostatitis/chronic pelvic pain syndrome. Chin Med J (Engl) 2011;124:514-518.

20. Nickel JC. Understanding chronic prostatitis/chronic pelvic pain syndrome (CP/CPPS). World J Urol 2013;31:709-710.

21. Krannich M, Rief W, Martin A, Brahler E, Mewes R, Glaesmer H. [How do somatoform and depressive symptoms and syndromes affect life satisfaction? Results from a representative population survey in Germany]. Psychother Psychosom Med Psychol 2013;63:217-224.

22. Pae CU. Critics on "adjunctive memantine therapy for cognitive impairment in chronic schizophrenia: a placebo-controlled pilot study". Psychiatry Investig 2013;10:98-99. 